\title{
Incorporating Empathic Responses into Postpartum Depression Psychotherapy
}

\author{
Yumei Li \\ Higher Education Press, Beijing, China \\ Email: bellalicn2018@gmail.com
}

How to cite this paper: Li, Y. M. (2021). Incorporating Empathic Responses into Postpartum Depression Psychotherapy. Open Journal of Depression, 10, 1-13. https://doi.org/10.4236/ojd.2021.101001

Received: October 25, 2020

Accepted: February 7, 2021

Published: February 10, 2021

Copyright (C 2021 by author(s) and Scientific Research Publishing Inc. This work is licensed under the Creative Commons Attribution International License (CC BY 4.0).

http://creativecommons.org/licenses/by/4.0/

\begin{abstract}
This study aims to delve into linguistic evidence for therapists to achieve better therapeutic outcomes by empowering women suffering from postpartum depression with empathic responses. Conversation samples are transcribed from videos for analysis. The focus of analysis is on language features of two postpartum women based on the Postpartum Voice of Depression Response Model. Expressions of negative feelings fall into four emotional domains. Language features of negative feelings are salient in postpartum women, often represented by a frequent use of negative words and sustaining on a sad topic. In clinical settings, therapists can help postpartum women get out of disturbing states by means of eliciting their inner feelings, identifying negative expressions and offering empathic responses accordingly. An empathic attitude matters in a person-centered clinical setting, which means a reassuring expression from the therapist can ease anxiety of the patient. The application of the Model has implications for emotional support in clinical settings, particularly in terms of differentiating between four emotional domains and showing understandings of negative feelings experienced by postpartum women.
\end{abstract}

\section{Keywords}

Postpartum Depression, Vulnerability, Psychotherapy, Language Features, Emotional Domains, Empathic Responses

\section{Introduction}

The symptoms of anxiety and depression are common for women during the perinatal period; statistics show that up to one out of six women experience postpartum depression in Australia (BeyondBlue, 2019). A substantial body of literature shows that women who suffer from postpartum depression (or postnatal depression) describe this period as "the darkest days of life", and the de- 
pression highlights a strong sense of powerlessness, anxiety, anger or grief (Aiken, 2000; Fettling \& Tune, 2005; Moritz, Kelly, Xu, Toews, \& Rickhi, 2011; Hodson, 2014; Odessa, 2013). In particular, this paper focuses on psychotherapy of postpartum depression with regard to the specific methods of empowering women with empathic responses. Literature suggests empathic interventions have been used as a common strategy to address the issues of depression, the effectiveness of which manifest in a variety of perspectives. Specifically, empathic responses can have a direct impact on patients' self-esteem, confidence and self-identity (Fitzgerald, 2013; HealthEngine, 2015; Kleiman, 2017; Pounds, 2010). However, Kleiman (2017) points out that in a large number of clinical cases the patients report that "their health practitioners are patronizing and misinformed" (p. 127). Thus, it is essential for therapists to take effective measures on empathic interventions for better therapeutic outcomes such as an intervention on the early stages of vulnerability, building a closer therapeutic relationship and mitigating stress and anxiety (Fitzgerald, 2013; Kleiman, 2017).

To achieve better therapeutic outcomes, it is important for therapists to recognize the most salient symptom of a postpartum woman's depression. A holding intervention without regard to the salient features of the postpartum woman's speech will be of little avail. Imagine that on the surface, a therapist is trying to assure a postpartum woman with empathic responses, however, it is not enough for a woman with depression to hear utterances such as "it's going to be better for you" and "I understand your situation". If the therapist delves into the underlying reasons in greater depth, he/she may find out the causes for the loss of self and further develop a set of strategies to help the individual cope with the issues.

This paper delves into the analysis of the most prominent emotional domains of two postpartum women based on the Postpartum Voice of Depression Response Model. The response to each utterance is presented for researchers and therapists to consider. Prior to the analysis, the key constructs in relation to the language features of depression will be introduced. The four sets of emotional domains will be elaborated. In the following section, the analysis will be focused on specific emotional domains experienced by the two postpartum women. Based on the most salient language features, the most typical depression symptoms will be recognized. The suggested responses will be given to help ease the depression symptoms. The analysis has implications for empowering postpartum women with the most effective holding statements.

\section{Language Features of Postpartum Depression}

\subsection{Affect, Negative Words and Stressed Intonation}

Fine (2006) identifies three types of language features signaling mood disorders such as depression and mania:

1) Affect ${ }^{1}$ that suggests the feelings of the speaker.

2) The changes in topics, predominantly conveying negative thoughts in de-

${ }^{1}$ Affect in this context refers to a construct within the framework of Systemic Functional Linguistics. It represents a main type of attitude that serves the interpersonal metafunction (Martin \& Rose, 2007). 
pression.

3) The phonological feature of language, particularly "slowed and decreased volume of speech in depression" (Fine, 2006: p. 237).

The three types of general language features manifest in different ways. People with depression may make statements about their feelings such as hopelessness, sadness or anxiety. These subjective reports are often realized by verbs connecting an emotion to an individual such as see, feel and think, which are categorized into three major types, known as "perception" (see/hear), "affect" (feel) and "cognition"(think/know/understand) (Fine, 2006: p. 238). In particular, verbs of sensing (feel) can be followed by an adjective (sad/horrible), indicating the mood of the speaker. Note that the speaker can not only express mood directly by saying "I feel sad" but also talk indirectly about his/her feelings or perceptions with facial expressions or body language.

Initiating and sustaining a sad topic is typical in a conversation of a speaker with depression. Although referring to a sad topic does not necessarily indicate a depressed mood, the frequent mentions of sad topics may signal sadness and hopelessness. A person with depressed mood may use negative utterances frequently. Fine (2006) exemplifies this salient language feature with frequent uses of " $n$ 't" and "no" in a depressed speaker's speech. He further points out that frequent negative markers could be accompanied with non-verbal signs of depression. All these signs represent diminished interest or pleasure in everyday activities. Of particular concern to the clinician is when a hobby becomes uninteresting to the speaker. Thus, less enthusiasm on once favored topics is an important indicator of depression.

Another type of language feature of depressed people is related to intonation or volume of speech. A person with depression may be uninterested, detached or depressed in the communication, whose expressions are characterized by a low level of engagement in the interaction and a lack of emotional involvement with conversation partners. Normally people show their interest in a certain topic by stressing particular words. The rise or fall in pitch signals interaction between the speaker and the listener. Nevertheless, a depressed person's speech contrasts sharply with the normal changes of intonation. It is worth mentioning that a combination of the three types of linguistic features signals symptoms of mood disorders including irritability and depressed mood.

\subsection{Four Emotional Domains}

Kleiman (2017) focuses on emotional expressions of postpartum depression and particularly examines empathic responses in emotional involvement, incorporating the Postpartum Voice of Depression Response Model into analysis. The Model comprises four sets of emotional domains, which are " 1 ) powerlessness/confusion, 2) sorrow/grief, 3) fear/anxiety, and 4) shame/dependence" (Kleiman, 2017: p. 116). Kleiman (2017) points out that the Model enables a postpartum woman to "feel held and cared for" (p. 117) and to know whether the emotional states are within the normal range. It is significant for a postpartum 
woman to get disturbing states validated at an early stage because an early validation can help reduce her symptoms from the outset (Kleiman, 2017). Predominantly, Kleiman (2017) points out that the intense vulnerability can be dealt with specific techniques.

\section{Conceptual Framework}

\subsection{Empathy}

Fitzgerald (2013) explains that empathy refers to a therapist's attempt to understand the patient's experience. Pounds (2010) points out that studies demonstrate empathy allows patients to feel free to talk about their conditions, leading to clearer diagnoses, higher patient satisfaction and better therapeutic outcomes.

\subsection{Psychotherapy}

Psychotherapy refers to a professional counselling process, the focus of which is often on an individual issue, mood disorder or emotional suffering (HealthEngine, 2015). It is complex due to diverse types of psychotherapy practices. Psychotherapy can work through language analysis, reflection, detailed discussion and developing communication skills (HealthEngine, 2015). The aim of psychotherapy is to enact understanding of a person's experience, behaviour and ultimately to investigate the contributors to the existing psychological issues (HealthEngine, 2015).

\subsection{Empathic Responses}

Fitzgerald (2013) defines an empathic response as a term specifically used in person-centered clinical settings that shows an empathic attitude of a therapist towards the client. An empathic response can be used as a means of communication. The ultimate goal of using an empathic response is to show understanding of the client's inner feelings and to examine how these feelings affect or reflect his/her current experience (Fitzgerald, 2013).

\subsection{The Postpartum Depression}

The postpartum depression, or postnatal depression, refers to a series of depressive symptoms representing emotional or behavioural disorders (Kleiman, 2017). According to BeyondBlue (2019), the Edinburgh Postnatal Depression Scale (EPDS) provides a checklist consisting of a set of questions that guide both the new mothers and professionals to validate the disturbing states. The questions involve whether the surveyee

1) being able to see "the funny side of things" and look forward to things with enjoyment;

2) blaming herself unnecessarily when things go wrong;

3) being anxious or worried, feeling scared or panicky for no good reason;

4) having the ability to cope with things;

5) having difficulty sleeping; or 
6) feeling sad or miserable, crying or having the thought of harming oneself.

\section{The Postpartum Voice of Depression Response Model}

The Model has been developed based on clinical observations. As mentioned above, the Model comprises four distinctive emotional domains. Emotions of all the domains tend to result in a sense of anguish. A therapist can validate a postpartum woman's disturbing states by differentiating between different sets of domains. The Model can help the therapist appropriately respond to utterances of different domains (Table 1).

\subsection{Domain A: Powerlessness/Confusion}

Domain A is characterized by powerlessness. Symptoms such as feeling helpless, defenceless or feeling out of control belong to Domain A (Kleiman, 2017). Kleiman (2017) emphasizes that feeling powerless tends to exacerbate postpartum women's current state of distress, and further explains that an optimal response to powerlessness can be an expression of consistent encouragement and reassurance. This kind of empowerment can "infuse an early sense of hopefulness" (Kleiman, 2017: p. 119). Additionally, words of reassurance with an appropriate tone indicate kindness of the therapist. A reassuring expression is often used as a means of empowerment for the treatment of postpartum women.

\subsection{Domain B: Sorrow/Grief}

Domain B features a postpartum woman's state of the loss of self that tends to lead to other domains. Kleiman (2017) points out that grief, a primary emotional state, is represented by the predominant loss of self. It is prominent that the state of grief extends beyond and infiltrates other domains. For those postpartum women who suffer from infant loss or pregnancy loss, bereavement is a major contributor to grief; for those who give birth to healthy babies, the main causes are often discontent or denial. This means factors other than infant loss or pregnancy loss can also lead to a sense of sorrow or grief. Kleiman (2017) states that "loss pervades the postpartum milieu" (p. 120). She further points out that any sense of loss could complicate emotions. If a therapist steers a postpartum

Table 1. Postpartum depression symptoms based on the Voice of Depression Response Model.

\begin{tabular}{ccc}
\hline Domain & Features & Engaged empathy \\
\hline Domain A & Powerlessness/confusion & Reassurance \\
Domain B & Sorrow/grief & Hope \\
Domain C & Fear/anxiety & Control \\
Domain D & Shame/dependence & Nurturing \\
\hline
\end{tabular}

Source: This table shows the four major domains of postpartum depression symptoms according to the Model established by the Postpartum Stress Center, LLC (Kleiman, 2017: p. 118). In therapeutical settings, the therapist can make empathic responses to negative utterances to ease the depression symptoms. 
woman in the direction of positivity and empowers her with hope, she is likely to step beyond the pervasive negativity. The best response to a grief expression is to acknowledge feeling sad is natural in such a period, which can mitigate a sense of guilt over a perception of unfounded grief. It is essential to empower a postpartum woman with a sense of hope. Without hope, there would be no such holding techniques that can work on a person with depression.

\subsection{Domain C: Fear/Anxiety}

Domain C is represented by anxiety, "a hallmark symptom of postpartum depression" (Kleiman, 2017: p. 122), which is a distinctive feature from other types of depression. The complexity of anxiety is in varying forms such as excessive worry and panic disorder. The common feelings involve agitation, obsessive thinking and panic attacks. Different postpartum women experience different symptoms, thus, "a full-blown diagnosis is not always applicable" (Kleiman, 2017: p. 122). Postpartum women tend to ascribe the feeling of anxiety to a loss of control or awful thoughts. Kleiman (2017) points out that the expert response to anxiety is to give a steady encouragement to a postpartum woman so that she can deal with an unpredictable situation with patience and attention.

\subsection{Domain D: Shame/Dependence}

Domain D has to do with a sense of dependency and shame induced by powerlessness. Kleiman (2017) states that postpartum women tend to feel guilty and needy when they are overwhelmed by a feeling of powerlessness, while feeling dependent in turn tends to put them in a disagreeable position of asking for help. Notably, there is a challenge to postpartum women that there is a sharp contrast between their anticipation of what would happen and their state of being (Kleiman, 2017). The loss of control and the situation of having to seek assistance give rise to the sense of powerlessness, which ultimately result in a failure in withstanding the pressures and the reluctance to seek help (Kleiman, 2017). Kleiman (2017) further points out that in clinical settings, the presence of shame tends to prevent the postpartum woman from disclosing her situation to the therapist, and the entrenchment of the shame can hinder intervention. Kleiman (2017) explains that the best response to dependency and shame is nurturance as offering nurturance will help ease the shame. The encouragement and guidance from the therapist will enable the postpartum woman to develop a greater tolerance to the state of being. It is essential that the postpartum woman accept the emotional support. In practice, the compassion can be expressed by words, or sometimes it can be shown in a way of keeping silence. It is essential to the therapeutic outcomes that a bond between the therapist and the postpartum woman be established. Thus, it could be concluded that the collaborative rapport, or the essence of relationship, allows the holding process of dependency to take place.

Prominently, Kleiman (2017) points out that in clinical cases some specific 
feelings belong to more than one domain. For example, anger could be interpreted as belonging to any of the four domains or even all the domains, thus, the response is contingent on the specific symptom or the circumstance. In addition, Kleiman (2017) explains that "the domains are not mutually exclusive" and "holding points overlap" (p. 127). This means that there are blurred boundaries between domains.

\section{Implementation of the Model}

\subsection{Instance 1}

The conversation is extracted from a video file retrieved from the website of YouTube. The title of the film is "A New Life. Postpartum Depression" (see transcripts below). Analysis will be focused on the language features of the postpartum woman Michelle, especially on the emotional expressions she uses when she loses patience with the baby's constant crying and bursts into tears. She then discloses her vulnerability to her neighbour and describes her suffering. The analysis will look at the way she describes her feelings and the causes of her depression symptoms. In particular, the expressions will be categorized into specific domains. The possible response to each set of expression will be provided from a therapist's perspective.

Neighbour. Michelle, you can talk to me.

Michelle: It is all my fault. Suddenly he starts crying and won't stop. He refuses to be in my breast. And my husband takes him and says he is not grown enough. I feel horrible. It looks like everybody can take better care of him than me. He says I can take good care of him easier. It's not even about him. It's about me. I m enough for him, but at the same time I just want to run away.

Michelle, the postpartum woman in this movie, often feels exhausted after giving birth to a baby. The analysis in Table 2 will focus on the conversation between Michelle and her neighbour, another young woman who shares her

Table 2. An analysis of the emotional expressions

\begin{tabular}{|c|c|}
\hline Expression & Domain \\
\hline It is all my fault. & $\begin{array}{l}\text { Domain A: powerlessness/confusion; } \\
\text { Domain D: shame/dependence }\end{array}$ \\
\hline $\begin{array}{l}\text { Suddenly he starts crying and won't stop. He refuses to be in } \\
\text { my breast. }\end{array}$ & $\begin{array}{l}\text { Domain A: powerlessness/confusion; } \\
\text { Domain C: fear/anxiety }\end{array}$ \\
\hline $\begin{array}{l}\text { And my husband takes him and says he is not grown } \\
\text { enough. }\end{array}$ & Domain A: powerlessness/confusion \\
\hline I feel horrible. & Domain B: sorrow/grief \\
\hline It looks like everybody can take better care of him than me. & Domain D: shame/dependence \\
\hline He says I can take good care of him easier. & Domain D: shame/dependence \\
\hline It's not even about him. It's about me. & Domain A: powerlessness/confusion \\
\hline $\begin{array}{l}\text { I m enough for him, but at the same time I just want to run } \\
\text { away. }\end{array}$ & Domain C: fear/anxiety \\
\hline
\end{tabular}


feelings about the difficulty in taking care of a new baby. When her neighbour tries to comfort her, Michelle describes how she feels about taking care of the baby and how her husband's attitude towards her and other family members' behaviour affect her feelings. Michelle's description features emotions of all the domains in the Model. For example, when she says, "it is all my fault", she reveals a sense of shame and low self-esteem under this circumstance (Domain D, shame/dependence), meanwhile this utterance also indicates a feeling of powerlessness. We can see her unspoken need that "it is all my fault, but I can do nothing about it. I need help. I need support". When she says, "suddenly he (the baby) starts crying and won't stop-he refuses to be in my breast", she implies a feeling of anxiety, and she feels a loss of control with the situation. Michelle also uses explicit expressions such as "I feel horrible" and "I just want to run away". The word "horrible" suggests a deep sorrow, while the intention "to run away" shows her fear and anxiety towards her suffering. The self-denial utterance that "everybody can take better care of him than me" could be the cause for her grief. Based on this kind of self-denial, she seems to be obsessed with a sense of sadness. Then the sadness leads to symptoms of other domains such as "it's all my fault" and "I just want to run away". Domain C (fear/anxiety) is manifested by an inclination to withdrawal and isolation ("to run away"). It is noticeable that Michelle uses a variety of negative expressions to describe qualities and events such as "all my fault", "refuse" and "horrible".

Despite Michelle's description features all four sets of domains, it seems that Domain A (powerlessness/confusion) and Domain D (shame/dependence) take precedence over others, thus she is in need of reassurance and nurturance. An expert emotional support from a therapist will help her regain confidence. In order to ease Michelle's sense of powerlessness and shame, I have designed a dialogue between a therapist and Michelle so that a steady encouragement and words of optimism will help her feel better.

Michelle: It is all my fault.

Therapist. I can see this feels terrible for you. Let's start with what troubles you most.

Michelle: Suddenly he starts crying and won't stop. He refuses to be in my breast. And my husband takes him and says he is not grown enough.

Therapist. Have you ever sought another mums' advice? She might explain why a baby is constantly crying. Also, you can check with the baby and see if he is hungry or he needs other help. Once you realize what causes the crying, you will feel better. Because you know what you could do about it.

Michelle: I feel horrible.

Therapist. I know it's hard.

Michelle: It looks like everybody can take better care of him than me. He says I can take good care of him easier.

Therapist. It must be uncomfortable for you. If you try setting limits with family, you can avoid such an overwhelming feeling.

Michelle: It's not even about him. It's about me. 
Therapist. I know you find it difficult to enjoy yourself in such an occasion. It will happen when you have to take care of a baby.

Michelle: $P m$ enough for him, but at the same time I just want to run away.

Therapist. I understand you don't feel like taking care of the baby right now, but we can take some steps, say, if you have a good rest, you might find that it is not that difficult to do something. Can you tell me how you have dealt with the stress before giving birth to the baby?

\subsection{Instance 2}

The information is also selected from a video file retrieved from YouTube, posted by the organisation of National Institute of Mental Health (NIMH) in the United States. The title is “Baby blues' - or Postpartum Depression?" (the monologue is presented below).

You know, I was a mess. I was crying. You know, I just could not seem to shake that sort of overwhelming feeling that I had. I think I am always worried about something happening to my babies or me. You know, it was constantly in my head that I couldn't relax. I was very wound up. I was just constantly checking on them.

This video aims to distinguish postpartum depression from baby blues, the symptoms of which approximately $80 \%$ of the new mothers report to have experienced. An expert explains that postpartum women often report a variety of symptoms involving ruminations, obsessions, anxiety and sleeping disorders. In this video, the postpartum woman recalls her experience of depression and elaborates on her feelings and behaviours at that time. The focus of analysis will be on possible responses to her emotions on the assumption that she had been communicating with a therapist in a psychotherapy setting. To identify the specific domains of the emotional expressions, I will use the Model as the analytical tool.

In this case, the postpartum woman describes anxiety and sadness she experienced after the babies were born. She talks about her feelings using negative utterances such as "I was a mess", "I was crying", "I was always worried about something" and "I was very wound up". The transcripts of the extracted monologue and a detailed analysis of her emotional expressions are presented in Table 3. As shown in the table, the most prominent language feature of the postpartum woman is anxiety. Most of the explicit negative expressions suggest an overwhelming sense of anxiety. Language features of anxiety manifest in a variety of expressions such as "overwhelming feeling", "worry about something", "couldn't relax" and "constantly checking on them". In addition, the words "always", "constantly" and "very" are used as intensifiers to stress the severity of her anxiety.

There is a correlation between different domains. For example, the utterance "I was a mess" indicates her state of being disturbed (Domain C, fear/anxiety), leading to a sense of powerlessness (Domain A, powerlessness/confusion). The utterance "I was crying" suggests she was overwhelmed by a sense of sorrow (Domain B, sorrow/grief). The sense of grief presumably originates from her 
Table 3. An analysis of the emotional domains.

\begin{tabular}{|c|c|}
\hline Expression & Domain \\
\hline You know, I was a mess. & $\begin{array}{l}\text { Domain A: powerlessness/confusion; } \\
\text { Domain C: fear/anxiety }\end{array}$ \\
\hline I was crying. & $\begin{array}{l}\text { Domain A: powerlessness/confusion; } \\
\text { Domain B: sorrow/grief }\end{array}$ \\
\hline $\begin{array}{l}\text { You know, I just could not seem to shake that sort of } \\
\text { overwhelming feeling that I had. }\end{array}$ & Domain A: powerlessness/confusion \\
\hline $\begin{array}{l}\text { I think I am always worried about something happening to } \\
\text { my babies or me. }\end{array}$ & Domain C: fear/anxiety \\
\hline You know, it was constantly in my head that I couldn't relax. & Domain C: fear/anxiety \\
\hline I was very wound up. I was just constantly checking on them. & Domain C: fear/anxiety \\
\hline
\end{tabular}

feelings of loss of self. The sense of loss could be assumed to be associated with the lack of control over the situation. The linguistic evidence is the utterance "I just could not seem to shake that sort of overwhelming feeling that I had" (Domain A, powerlessness).

As mentioned above, the solution to the issues has been designed on the assumption that the woman was consulting a therapist. The responses from the therapist will help ease her anxiety and support her with reassurance.

Woman: You know, I was a mess. I was crying.

Therapist. I am sorry you feel sad. It's okay to feel this way. You have two little babies to take care of. It is hard, but we can do something that can help you feel better.

Woman: You know, I just could not seem to shake that sort of overwhelming feeling that I had.

Therapist. What worries you most? Can you tell me more about your symptoms so that we can come up with a plan?

Woman: I think I am always worried about something happening to my babies or me. You know, it was constantly in my head that I couldn't relax.

Therapist. You are sharing your story. I can see you are taking steps to improve the situation. Post-partum depression is treatable. It can be treated by psychotherapy or by medication. You will feel better if we go forward.

Woman: I was very wound up. I was just constantly checking on them.

Therapist. I can imagine how anxious you have been. It is okay to be anxious soon after the babies were born. You can heal yourself over time. We can make up a plan to ease your anxiety.

The postpartum women in the two chosen cases seem to share a sense of powerlessness. Both of them are seeking help because they find themselves in a position of helplessness. They are wondering what they could do to address the issues. Their state of being could be supported by the therapists' reassurance. However, the two postpartum women seem to have distinctive types of main symptoms. Michelle has a sense of low self-esteem, while the mother of two new 
babies, in the second case, shows an overwhelming sense of anxiety. It is worth pointing out that some expressions belong to more than one domain. For example, when the mum, the character of the monologue, states that "I was a mess" and "I was crying", it suggests that she is in a state of stress and she is feeling out of control. It could be interpreted as a feeling of powerlessness, or it could be sadness. However, the main cause is the overwhelming anxiety. From this perspective, we can notice a blurred boundary between the domains. This finding is in line with the explanation that "some symptoms can be interpreted as belonging to more than one domain" (Kleiman, 2017: p. 117). Based on the domain analysis, specific empathic interventions have been given to ease their symptoms of stress. The limitation is that the effectiveness should be assessed based upon the actual therapeutic outcomes, and in practice, it still takes time to evaluate whether they are effective.

\subsection{A Summary Analysis}

If we compare the results of analysis between the two cases, we may find that the prominent language feature of the first case distinguishes from the second one: Michelle needs more encouragement and assertiveness, while the other needs to find out ways to relax. The positive side is that the two postpartum women are both crying out for help. The key problem is they are both unaware of their specific needs. If the therapists can understand the causes for their vulnerability, they can better assure them with optimal responses to attend to their needs. As Michelle shows more symptoms of shame and dependence, we can tell she is in urgent need of nurturance and confidence. In the second case, the woman's anxiety contrasts significantly with Michelle's dependence, thus, we can see that she needs a sense of ease. The therapist can not only help her stop the obsessive thinking but also help her find out what triggers her panic attack. It is important for the therapist to identify the most typical symptoms and provide optimal holding responses according to the postpartum women's own expressions.

\section{Implications}

In light of the analysis of the two cases, future research can be focused on the most salient language feature of postpartum women and the specific holding techniques. Engaged empathy is key to the advancement in psychotherapeutic outcomes. In therapeutic settings, the therapist can make an impact on the way how a postpartum woman perceives the world around her. The holding intervention from the therapist can also affect how the postpartum woman perceives herself. In other words, the focus of empowerment is on how to cope with grief and loss. A woman with low self-esteem may be in need of support from other people, especially on confidence building, while another woman showing severe symptoms of anxiety may not know she needs to find out ways to calm down. Based on the analysis in this study, the whole process of holding intervention in future research can be divided into four stages: 1) In the initial stage, it is essen- 
tial to ensure the postpartum woman is willing to cry out for help. 2) The therapist needs to identify the most typical symptom as different individuals are in need of different kinds of support. 3) The therapist can empower them with holding statements. 4) To evaluate the effectiveness of holding techniques, the therapist can observe or record their performance over a long period. It is significant to examine the effectiveness from the perspective of the postpartum women. For example, their voice can be recorded in a longitudinal study, preferably over a period of one year.

\section{Conclusion}

Studies show that a large number of postpartum women experience the similar suffering, and psychotherapy has been demonstrated to be effective in helping them out. However, in practice, different therapists tend to use different strategies when comforting postpartum women, thus, there have been varying degrees of effectiveness in the evaluation of therapeutic outcomes. Therapists need to evaluate specific issues that challenge postpartum women so that they can work out effective plans and give optimal responses. Through analysis, the Postpartum Voice of Depression Response Model has been justified as an effective tool to identify specific negative feelings the postpartum women are experiencing. Furthermore, the suggested responses are domain-specific so that the postpartum women can recognise their symptoms and open up to talk more about their feelings, or even get to know how they can heal themselves. Future research can be focused on the effectiveness of the holding responses by either observing the behaviours of the postpartum women or interviewing them for an evaluation of the methods.

\section{Acknowledgements}

I would like to express my sincere gratitude to my research supervisor, Dr. Yeagan Doran, the University of Sydney, for giving his support on organising the ideas and developing the structure of writing and for providing invaluable guidance on my research work throughout my postgraduate studies. His expertise and passion in research work have greatly inspired me. I would also like to thank him for his kindness and sense of humour.

\section{Conflicts of Interest}

The author declares no conflicts of interest regarding the publication of this paper.

\section{References}

Aiken, C. (2000). Surviving Post-Natal Depression: At Home, No One Hears You Scream. London, UK: Jessica Kingsley Publishers.

BeyondBlue (2019). Mental Health Checklist for Mums.

https://healthyfamilies.beyondblue.org.au/pregnancy-and-new-parents/maternal-ment 
al-health-and-wellbeing/mental-health-checklist-for-mums

Fettling, L., \& Tune, B. (2005). Women's Experience of Postnatal Depression: Kitchen Table Conversations. Melbourne, Australia: IP Communications.

Fine, J. (2006). Language in Psychiatry: A Handbook of Clinical Practice. London, UK: Equinox Pub.

Fitzgerald, P. (2013). Therapy Talk: Conversation Analysis in Practice. Basingstoke, UK: Palgrave Macmillan. https://doi.org/10.1057/9781137329530

HealthEngine (2015). Psychotherapy for Depression: Dr Jan Resnick. https://healthengine.com.au/info/psychotherapy-for-depression

Hodson, K. (2014, May 8). How to Tell the Difference between the Baby Blues vs. Postpartum Depression. Video File. https://www.youtube.com/watch?v=SXxjqRAf-zM

Kleiman, K. (2017). The Art of Holding in Therapy an Essential Intervention for Postpartum Depression and Anxiety. New York, US: Routledge. https://doi.org/10.4324/9781315696119

Martin, J., \& Rose, D. (2007). Working with Discourse: Meaning beyond the Clause (2nd ed.). London, UK: Continuum.

Moritz, S., Kelly, M., Xu, T., Toews, J., \& Rickhi, B. (2011). A Spirituality Teaching Program for Depression: Qualitative Findings on Cognitive and Emotional Change. Complementary Therapies in Medicine, 19, 201-207. https://doi.org/10.1016/j.ctim.2011.05.006

Odessa, M. (2013, October 14). Instructional Videos for Moms-Baby Blues vs. Post-Partum Depression. Video File. https://www.youtube.com/watch?v=WmCaYFPASBk

Pounds, G. (2010). Empathy as "Appraisal”: A New Language-Based Approach to the Exploration of Clinical Empathy. Journal of Applied Linguistics and Professional Practice, 7, 140-162. https://doi.org/10.1558/japl.v7i2.145 